\title{
CFTR: cystic fibrosis and beyond
}

\author{
Marcus A. Mall ${ }^{1,2}$ and Dominik Hartl ${ }^{3}$
}

\begin{abstract}
Affiliations:
'Dept of Translational Pulmonology, Translational Lung Research Center Heidelberg (TLRC), University of Heidelberg, Member of the German Center for Lung Research (DZL), Heidelberg, Germany.

${ }^{2}$ Division of Paediatric Pulmonology and Allergy and Cystic Fibrosis Center, Dept of Paediatrics, University of Heidelberg, Member of the German Center for Lung Research (DZL), Heidelberg, Germany.

${ }^{3}$ Paediatric Infectiology and Immunology, Dept of Pediatrics, University of Tübingen, Tübingen, Germany.
\end{abstract}

\section{Correspondence:}

Marcus A. Mall, Dept of Translational Pulmonology, Translational Lung Research Center Heidelberg (TLRC), University of Heidelberg, Im Neuenheimer Feld 350, 69120 Heidelberg, Germany.

E-mail: Marcus.Mallamed.uni-heidelberg.de

ABSTRACT Cystic fibrosis (CF) remains the most common fatal hereditary lung disease. The discovery of the cystic fibrosis transmembrane conductance regulator (CFTR) gene 25 years ago set the stage for: 1) unravelling the molecular and cellular basis of CF lung disease; 2) the generation of animal models to study in vivo pathogenesis; and 3) the development of mutation-specific therapies that are now becoming available for a subgroup of patients with CF. This article highlights major advances in our understanding of how CFTR dysfunction causes chronic mucus obstruction, neutrophilic inflammation and bacterial infection in CF airways. Furthermore, we focus on recent breakthroughs and remaining challenges of novel therapies targeting the basic CF defect, and discuss the next steps to be taken to make disease-modifying therapies available to a larger group of patients with CF, including those carrying the most common mutation $\triangle F 508$-CFTR. Finally, we will summarise emerging evidence indicating that acquired CFTR dysfunction may be implicated in the pathogenesis of chronic obstructive pulmonary disease, suggesting that lessons learned from CF may be applicable to common airway diseases associated with mucus plugging.

@ERSpublications

CFTR dysfunction causes CF and may be implicated in more common chronic obstructive lung diseases, such as COPD http://ow.ly/wplhu and the German Cystic Fibrosis Association Mukoviszidose e.V.

Conflict of interest: Disclosures can be found alongside the online version of this article at erj.ersjournals.com 


\section{CFTR, basic ion transport defects and cystic fibrosis lung disease}

Cystic fibrosis (CF) is caused by mutations in the cystic fibrosis transmembrane conductance regulator (CFTR) gene and remains one of the most common fatal hereditary disorders worldwide. Although CF is a complex multi-organ disease, morbidity and mortality are mainly determined by chronic obstructive lung disease that evolves from early onset mucus plugging in the small airways, chronic neutrophilic airway inflammation and bacterial infection. The discovery of the CFTR gene 25 years ago set the stage for unravelling the molecular and cellular basis of CF lung disease [1-3]. CFTR is a multi-domain membrane protein that belongs to the large family of adenine nucleotide-binding cassette transporters consisting of two transmembrane domains, two nucleotide binding domains (NBDs) and a unique regulatory domain [4]. It is expressed in the airway surface epithelium, submucosal glands and many other epithelial organs [5]. CFTR functions as an anion channel, which is regulated by protein kinase A-dependent phosphorylation of its regulatory domain and binding of ATP to the nucleotide binding domains, and conducts $\mathrm{Cl}^{-}$and bicarbonate. In the airways, CFTR also functions as a regulator of the amiloride-sensitive epithelial $\mathrm{Na}^{+}$channel $(\mathrm{ENaC})$ that constitutes the limiting pathway for $\mathrm{Na}^{+}$absorption [6-9]. As a consequence, mutations in CFTR cause a basic ion transport defect, which is characterised by deficient cyclic adenosine monophosphate-dependent anion secretion and enhanced $\mathrm{ENaC}$-mediated $\mathrm{Na}^{+}$absorption in CF airways (fig. 1) [10-12]. The most common mutation, a deletion of phenylalanine at position 508 $(\triangle \mathrm{F} 508)$ in NBD1, impairs protein folding, plasma membrane expression, function and stability of CFTR, and is present on at least one allele in $\sim 90 \%$ of patients with CF in Europe and North America [4]. In addition, CF can probably be caused by over 1900 other mutations in the CFTR gene (www.genet.sickkids. on.ca) with diverse molecular consequences ranging from defective synthesis, gating, conductance or reduced numbers of CFTR channels at the apical plasma membrane $[13,14]$. Of note, a recent study demonstrated that only $\sim 8 \%$ of the reported CFTR variants have an allele frequency of $\geqslant 0.01 \%$, whereas the others are extremely rare and their functional consequences remain largely unknown [15]. While a complete understanding of the link between CFTR dysfunction, impaired regulation of ion transport, and the complex in vivo pathogenesis of airway inflammation and impaired host defence is still missing, important progress has been achieved through translational studies in primary airway cultures from patients with CF and animal models that phenocopy CF lung disease [16-18].

\section{Airway surface dehydration: a key disease mechanism in CF lung disease}

These translational studies led to the current concept that dehydration of the thin film of liquid $(\sim 7 \mu \mathrm{m})$ that covers healthy airway surfaces is a key mechanism in initiating and promoting lung disease in CF. Because active ion transport is coupled with water flow, the basic defect causes an imbalance between secretion and absorption of the epithelial lining fluid rendering CF airway surfaces vulnerable to dehydration $[19,20]$. Studies in cultured primary airway epithelia from CF patients carrying a broad spectrum of CFTR mutations demonstrated that airway surface liquid (ASL) is depleted resulting in a collapse of the periciliary layer, including the cilia, and mucociliary dysfunction (fig. 1) [16]. However, the in vivo validation of these results was hampered by the fact that Cftr-deficient mice do not reproduce the basic CF ion transport defect in the airways, probably due to expression of alternative $\mathrm{Cl}^{-}$channels, and do not develop CF-like lung disease [21]. This limitation led to the generation of mice with airway-specific overexpression of $\mathrm{ENaC}(\beta \mathrm{ENaC}-\mathrm{Tg})$ to mimic enhanced $\mathrm{ENaC}$ activity $[17,22]$ and, more recently, the generation of CFTR-deficient pigs and ferrets [23-25] to test the in vivo consequences of imbalanced ion/ fluid secretion and absorption across CF airways. A series of studies in $\beta E N a C$-Tg mice validated the concept that ASL depletion with hyperconcentrated mucus impairs mucociliary clearance, causes CF-like mucus plugging and sets the stage for chronic airway inflammation and bacterial infection [22]. Interestingly, recent studies in $\beta \mathrm{ENaC}-\mathrm{Tg}$ mice maintained under germ-free conditions demonstrated that mucus stasis per se is sufficient to cause airway inflammation, probably due to trapping of inhaled irritants that trigger the release of pro-inflammatory chemokines, such as keratinocyte chemoattractant and macrophage inflammatory protein-2, from airway epithelia and/or macrophages, even in the absence of bacterial infection [26]. Conversely, in the presence of bacteria in the environment, $\beta E N a C-T g$ mice develop spontaneous airway infection in the neonatal period and show reduced clearance of CF-associated pathogens such as Pseudomonas aeruginosa and Haemophilus influenzae at older ages [17, 26]. Collectively, these studies demonstrated that this mouse model exhibits many characteristic features of early CF lung disease [27-29]. Of note, the lung disease phenotype of older $\beta \mathrm{ENaC}$-Tg mice also shows some differences compared to patients with $\mathrm{CF}$, such as transient eosinophilic inflammation, in addition to chronic airway neutrophilia at juvenile ages, and clearance of spontaneous bacterial airway infection and development of pulmonary emphysema rather than bronchiectasis in adult mice [26, 27, 30-33].

More recent studies in CFTR-deficient pigs and ferrets demonstrated that these large animal models also develop spontaneous CF-like lung diseases characterised by early onset mucus plugging, air trapping, 


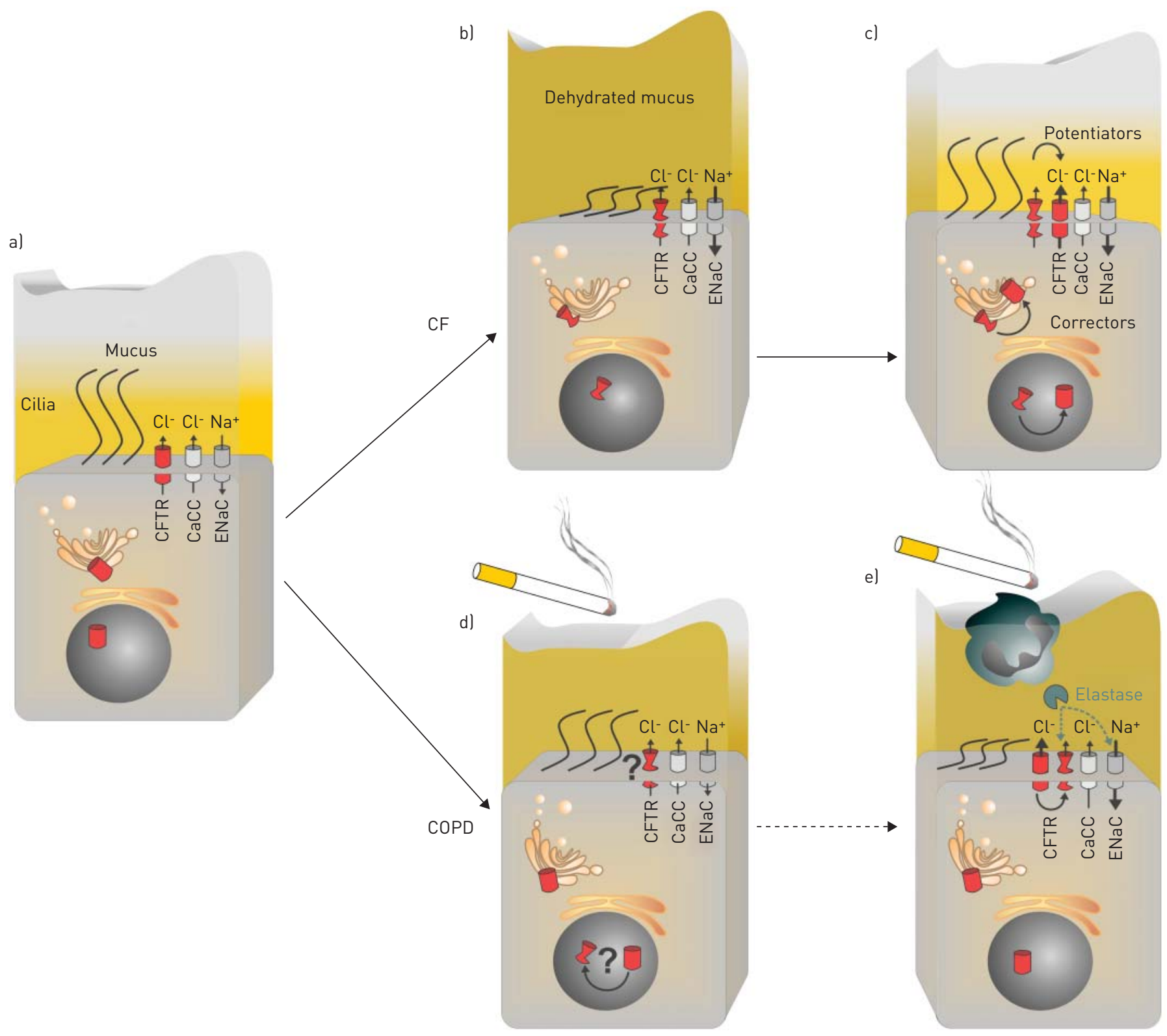

FIGURE 1 The role of the cystic fibrosis transmembrane conductance regulator (CFTR) in proper airway surface hydration and mucociliary clearance of normal airway epithelia, consequences of genetic and acquired CFTR dysfunction in patients with cystic fibrosis (CF) and chronic obstructive pulmonary disease (COPD), and therapeutic strategies to restore mutant CFTR function. a) In normal airway epithelial cells, CFTR is expressed at the apical membrane together with alternative $\mathrm{Ca}^{2+}$-activated $\mathrm{Cl}^{-}$channels $\left(\mathrm{CaCC}\right.$ ) and epithelial $\mathrm{Na}^{+}$channels (ENaC). CFTR-mediated secretion of $\mathrm{Cl}^{-}$and fluid, and concomitant inhibition of ENaC-mediated absorption plays a key role in proper hydration of the airway surface (periciliary layer and overlying mucus gel), which is essential for normal mucus clearance. b, c) In CF airway epithelia, mutation-specific defects cause deficient synthesis, processing, gating, conductance or reduced stability of $\mathrm{CFTR} \mathrm{Cl}^{-}$ channels (deformed channel). CFTR dysfunction at the apical membrane causes dehydration of the periciliary and mucus layers leading to mucociliary dysfunction and airway mucus plugging. Static airway mucus triggers inflammation and forms a nidus for bacterial infection (b). Current therapeutic strategies focus on the development of CFTR modulators that correct misprocessing of the most common mutation $\triangle$ F508 (correctors) and enhance the function of CFTR mutants that are inserted into the apical plasma membrane (potentiators) to restore airway surface hydration and mucus clearance (c). Alternative therapeutic approaches to improve airway surface hydration include inhibition of $\mathrm{ENaC}$ and activation of alternative $\mathrm{Cl}^{-}$channels (CaCC) (c). Recent studies demonstrated that cigarette smoke impairs CFTR expression at several levels, including transcription and protein stability, and that CFTR function is substantially reduced in the airways of patients with COPD. d) This "acquired" CFTR dysfunction may impede proper mucus hydration, especially in the presence of inflammationinduced goblet cell metaplasia and mucin hypersecretion, and put smokers at risk for mucus stasis and chronic airways disease. e) Proteases released in neutrophilic airway inflammation, such as neutrophil elastase, further degrade CFTR and activate ENaC by proteolytic cleavage, and thus aggravate airway surface dehydration and mucociliary dysfunction in CF and COPD. 
neutrophilic inflammation and bacterial infection of the respiratory tract $[18,34,35]$. Of note, bioelectric studies demonstrated deficient CFTR-mediated anion transport, but no increase in ENaC-mediated $\mathrm{Na}^{+}$ transport, in the lower airways of newborn CF pigs. Based on these results, the relative contribution of alterations in CFTR- versus ENaC-mediated ion transport in early CF lung disease has been discussed controversially [36]. However, the magnitude of ENaC-mediated $\mathrm{Na}^{+}$absorption in older CF pigs with chronic airway inflammation, i.e. under conditions when $\mathrm{ENaC}$ activity was found to be increased in native airway tissues from patients with CF in vitro and in vivo [10-12], remains unknown. Furthermore, studies on mucus concentration as a measure of airway surface hydration, as well as measurements of mucociliary clearance, are pending in CF pigs and ferrets. However, it was demonstrated that fluid secretion from submucosal glands is deficient in the CF pig model [37]. Taken together, the striking similarities between the early lung disease phenotypes of $\beta \mathrm{ENaC}$-Tg mice, CF pigs and $\mathrm{CF}$ ferrets indicate that airway surface dehydration caused by an imbalance between CFTR-mediated anion secretion and ENaC-mediated $\mathrm{Na}^{+}$ absorption by the surface epithelium of conducting airways and submucosal glands may play a central role in the in vivo pathogenesis of CF lung disease, which produces mucus stasis with airflow obstruction, triggers inflammation and provides a nidus for bacterial infection (fig. 1).

\section{Airway proteases aggravate basic CF ion transport defect}

Importantly, emerging evidence suggests that this pathogenetic sequence is not unidirectional, but that factors released in chronic neutrophilic inflammation can have profound effects on epithelial ion transport and aggravate airway surface dehydration in CF. For example, recent studies showed that neutrophil elastase $(\mathrm{NE})$, a major product released from activated neutrophils that has recently been identified as a key risk factor for the development of bronchiectasis in young children with CF diagnosed by newborn screening [38], is also a potent regulator of CFTR and ENaC. Besides its previously described roles in the modulation of airway inflammation, mucus hypersecretion, bacterial killing, and proteolytic lung and immune cell damage [32, 39-42], NE can activate ENaC by proteolytic cleavage and removal of "inhibitory" segments, probably leading to a conformational change of the channel that improves its conductivity for $\mathrm{Na}^{+}[43,44]$. Interestingly, a recent study showed that wild-type CFTR, but not $\Delta \mathrm{F} 508$, interacts with ENaC in the plasma membrane of airway epithelial cells and, thus, may impede the proteolytic stimulation of ENaC by $\mathrm{NE}$ and potentially other extracellular proteases released by inflammatory cells $[45,46]$. These results suggest that lack of this "anti-proteolytic" function of CFTR may contribute to increased $\mathrm{Na}^{+}$absorption in chronically inflamed CF airways, and may explain why ENaC-mediated $\mathrm{Na}^{+}$transport was not elevated in newborn $\mathrm{CF}$ pigs prior to the onset of chronic neutrophilic airway inflammation [36]. Furthermore, recent studies demonstrated that NE leads to degradation and loss of $\mathrm{CFTR} \mathrm{Cl}^{-}$channel function through a pathway involving the activation of intracellular calpains (fig. 1) [47]. Therefore, an imbalance between NE and potentially other proteases released from inflammatory cells or bacteria on the one hand, and protective anti-proteases on the other hand [44], may inevitably aggravate airway surface dehydration and also impede therapeutic approaches targeting the basic ion transport defect in CF airways once chronic inflammation and infection are established.

Potential role of airway surface dehydration in obstructive lung diseases beyond CF Beyond CF, emerging evidence from biophysical studies suggest that airway surface dehydration may also be implicated in the pathogenesis of other chronic airways diseases [48, 49]. These studies showed that an increase in the concentration of mucins (i.e. dehydration) in the mucus gel that covers airway surfaces results in an increase in its osmotic pressure. Above a critical threshold, the osmotic modulus of the dehydrated mucus layer exceeds the osmotic modulus of the periciliary layer. This leads to compression of the subjacent periciliary layer and cilia, and ultimately mucociliary dysfunction [48]. As mucus hyperconcentration not only results from dysregulated epithelial ion and fluid transport as in CF, but also from increased mucin secretion driven by chronic airway inflammation and infection [39, 50], these results suggest that airway surface dehydration may be a common disease mechanism that contributes to the pathogenesis of a broad spectrum of mucostatic airway diseases including asthma and chronic obstructive pulmonary disease (COPD) $[20,49]$. This concept is supported by recent studies in mice with experimental asthma demonstrating that T-helper cell type 2-induced mucus hypersecretion is associated with a hypersecretory ion transport phenotype that protects "asthmatic" wild-type mice from airway mucus obstruction in vivo, whereas mice that lack the epithelial $\mathrm{Cl}^{-}$channel SLC26A9 (solute carrier family 26 member 9), a member of the SLC26 family of anion transporters that is strongly expressed in the lung [51], develop airway mucus obstruction due to a reduced capacity to increase $\mathrm{Cl}^{-}$and fluid secretion in parallel to mucin hypersecretion in allergic airway disease $[52,53]$.

\section{Lack of bicarbonate impairs mucus formation and bacterial killing in CF airways}

In addition to airway surface dehydration, emerging evidence suggests that deficient CFTR-mediated bicarbonate secretion plays an important role in the pathogenesis of mucus obstruction and impaired host 
defence in CF airways. Impaired bicarbonate secretion results in reduced pH of the ASL in CF [54]. Recent studies in the CF mouse intestine demonstrated that the presence of bicarbonate is crucial for normal unpacking and expansion of mucins when they are secreted from intracellular granula onto the intestinal surface $[55,56]$. These results led to the hypothesis that impaired bicarbonate secretion may also lead to the formation of aberrant mucus in the airways, and that this defect may contribute to increased mucus viscosity, adhesion and mucus stasis in CF [57].

Furthermore, recent studies in the CF pig model demonstrated that reduced airway surface $\mathrm{pH}$ impairs bacterial killing. CF pigs fail to eradicate bacteria from their lungs after birth [18]. By dissecting underlying mechanisms causing impaired anti-bacterial host defence, the reduced ASL pH in CF pigs was found to render the anti-microbial peptides, such as lysozyme and lactoferrin, less effective, which may be key to reduced elimination of inhaled or aspirated bacteria and bacterial colonisation of CF airways [34]. These results may have important therapeutic implications, as they predict that re-alkalinisation of the airways, for instance by aerosolised bicarbonate or rescue of mutant CFTR function, has the potential to restore antibacterial host defence and prevent chronic airway infection in CF.

\section{Emerging novel therapies to rescue mutant CFTR: breakthroughs and remaining challenges}

Since a complete review of CFTR replacement and pharmacotherapy approaches is beyond the scope of this article, we will focus on the recent major breakthroughs with therapies designed to rescue mutant CFTR function, and the remaining challenges that still have to be overcome to make these causal therapies available for most patients with $\mathrm{CF}$.

\section{High throughput screening led to the discovery of the first drug acting on mutant CFTR}

The discovery of CFTR was also the cornerstone for the development of drugs that rescue mutant CFTR function to provide a causal therapy for CF. However, early studies showed that a wide spectrum of CFTR mutations can cause distinct classes of CFTR dysfunction through different molecular mechanisms ranging from deficient protein translation (class I), processing (class II), gating (class III), conductance (class IV), and reduced synthesis or stability (class V) of CFTR $\mathrm{Cl}^{-}$channels $[13,14]$. These studies predicted that causal CF pharmacotherapy has to be mutation-specific or at least specific for a group of mutations that belong to a similar class of dysfunction. These insights, together with so far unsuccessful attempts to correct mutant CFTR by gene therapy [58], led to the development of high-throughput screening to utilise large chemical libraries for the discovery of drugs that rescue CFTR mutations that affect synthesis and processing ("correctors"), and drugs that improve deficient gating and/or conductance of mutant $\mathrm{CFTR}^{-} \mathrm{Cl}^{-}$channels that are inserted into the apical plasma membrane ("potentiators") (fig. 1) [59, 60]. This approach was highly successful and, so far, has led to several investigational drugs [61, 62], and the development of the CFTR potentiator ivacaftor (VX-770), which was shown to improve lung function and reduce pulmonary exacerbations in pivotal clinical trials. Ivacaftor was approved in 2012 as the first mutation-specific therapy for CF patients carrying the G551D gating mutation $[63,64]$.

The G551D mutation is expressed and inserted into the apical plasma membrane at normal levels; however, the ATP-induced dimerisation of the two NBDs required for normal gating of the CFTR channel is defective, resulting in a very low open probability [61]. Although its mechanism of action remains incompletely understood, functional studies at the single channel level demonstrated that ivacaftor improved gating and increased the open probability of the G551D channel $[61,65]$. In cultured human CF bronchial epithelial cells, this effect rescued G551D-mediated $\mathrm{Cl}^{-}$transport to $\sim 50 \%$ of wild-type CFTR function, and reduced increased $\mathrm{ENaC}$-mediated $\mathrm{Na}^{+}$and fluid absorption [61]. In the first phase III study, conducted in adolescent and adult CF patients ( $\geqslant 12$ years of age), ivacaftor improved the forced expiratory volume in $1 \mathrm{~s}\left(\mathrm{FEV}_{1}\right) \%$ predicted by $10.6 \%$ and reduced the likelihood of pulmonary exacerbation by $55 \%$. Therapeutic effects on lung function were consistently observed by 2 weeks after starting therapy and maintained to week 48 . Of note, $75 \%$ of patients in this age group showed an improvement in FEV1 \% pred by $\geqslant 5 \%$ and therapeutic effects on lung function were independent from baseline FEV1, age or sex [63]. Similar effects with an overall improvement of FEV1 \% pred of $12.5 \%$ were observed in younger CF patients (6-11 years of age) with a G551D mutation, and it was shown that ivacaftor improves the lung clearance index in children with CF with normal spirometry $[64,66]$. While it remains to be seen to what extent these therapeutic benefits on pulmonary function are cumulative to already existing symptomatic therapies [67-70], this breakthrough provides an important proof-of-concept that mutation-specific therapy is possible and may, therefore, mark the beginning of a new era of personalised medicine for $\mathrm{CF}$, a topic that has been discussed in several recent reviews [71-73]. 
The CFTR potentiator creates opportunities for personalised medicine for CF

However, because the G551D mutation affects only 4\% of CF patients worldwide, considerable challenges remain that need to be overcome before a larger group of patients can benefit from these novel CFTR modulator therapies. In this context, recent in vitro studies demonstrated that ivacaftor can improve the open probability and, thus, potentiate other non-G551D gating mutations, as well as other rare CFTR mutations that cause mild defects in processing and/or conductance $[74,75]$. These results indicate that ivacaftor may be more broadly acting and beneficial for a larger group of CF patients that carry CFTR mutations with residual function, including pancreatic-sufficient patients. This hypothesis is currently being tested in a series of clinical trials in patients with non-G551D gating mutations and the pancreatic sufficient mutation $\mathrm{R} 117 \mathrm{H}$. If positive, ivacaftor may become available as an effective therapy for $\sim 15 \%$ to $20 \%$ of all patients with CF in the near future. Despite these advances, the functional consequences of many rare CFTR mutations, as well as their in vivo response to potentiator therapy, remain unknown [15]. Therefore, parallel development of sensitive functional assays for quantitative assessment of CFTR function at baseline and treatment responses will be important to identity individual CF patients with rare CFTR mutations who may benefit from ivacaftor or other CFTR modulators. In this context, recent studies demonstrated that $\beta$ adrenergic sweat secretion, as well as functional measurements in native intestinal tissues and intestinal organoids, are accurate and reliable assays to assess different levels of mutant CFTR function in individual patients with CF carrying a broad spectrum of mutations [76-79]. Therefore, these techniques may be helpful tools to improve our current understanding of how much CFTR activity is required to translate into a clinical benefit and facilitate implementation of truly personalised medicine for CF.

\section{Can CFTR modulator therapy prevent CF lung disease?}

Another important issue relates to the question of when ivacaftor therapy should be started in patients with responsive CFTR mutations in order to obtain maximal long-term benefits. It is generally assumed that CF lungs are structurally normal at birth and that lung disease progresses from potentially reversible abnormalities, such as airway mucus plugging and air trapping, to irreversible lung damage characterised by bronchiectasis and emphysema [28, 29, 38, 80, 81]. Importantly, recent studies in CF infants diagnosed by newborn screening indicate that irreversible lung damage may occur much earlier than expected, and mostly in the absence of respiratory symptoms. At the age of 3 months, a substantial fraction of CF infants already showed neutrophilic inflammation and structural lung damage, and these early abnormalities were a key risk factor for bronchiectasis in later life $[38,82]$. These results strongly suggest that early rescue of CFTR function may be essential to prevent and/or delay irreversible lung damage in CF. As sensitive noninvasive outcome measures for assessment of abnormal lung structure and function in infants and young children, such as computed tomography, magnetic resonance imaging and multiple breath washout, are becoming available [28, 38, 83-86], the ivacaftor clinical trial programme should be extended to studies in CF infants as soon as possible to exploit the narrow window of opportunity that has been created by widespread implementation of newborn screening and determine to what extent early therapy can delay or even prevent irreversible lung damage in CF.

\section{Correction of misprocessing of the most common mutation AF508 remains challenging}

In contrast to potentiation of CFTR mutants that are expressed in the plasma membrane, correction of mutations that impair CFTR synthesis or processing, including the most common mutation, $\Delta$ F508, remains a more challenging task. VX-809 (lumicaftor), the first CFTR corrector that was advanced to clinical development, restored $\Delta$ F508 surface expression and function up to $\sim 15 \%$ of wild-type CFTR in human bronchial epithelia cells in vitro [62]. In a recent phase II trial in CF patients homozygous for the $\Delta$ F508 mutation, treatment with VX-809 was well tolerated and caused a modest drop of sweat $\mathrm{Cl}^{-}$. However, there was no detectable improvement of CFTR function in the nasal epithelium as determined by nasal potential difference measurements, and no improvement in lung function or patient-reported outcomes [87]. These results suggest that VX-809 monotherapy may not be sufficient and that other approaches are required to rescue $\Delta \mathrm{F} 508$ function to a level that translates into clinical benefits for patients. One strategy that is already being tested in the clinical arena in phase III trials, including $\sim 1000 \Delta$ F508 homozygous CF patients, comprises the combination of VX-809 with ivacaftor in order to enhance both the number and open probability of $\Delta \mathrm{F} 508 \mathrm{Cl}^{-}$channels in the plasma membrane. In preclinical studies in human CF bronchial epithelial cells, this approach improved $\Delta$ F508-mediated $\mathrm{Cl}^{-}$transport up to $\sim 25 \%$ of wild-type CFTR when both compounds were added at the maximally effective concentrations [62]. The first results from the clinical corrector/potentiator combination trials are expected in 2014 and will provide important information of whether the level of functional improvement that can be reached with this combination therapy is sufficient to translate into clinical benefits for patients with the $\Delta \mathrm{F} 508$ mutation.

The second strategy relies on the identification of more efficacious next-generation correctors. This development is guided by insights from recent biochemical studies that may explain the mechanistic basis of 
the apparent efficacy ceiling of VX-809. These studies demonstrated that the $\Delta$ F508 mutation not only impairs folding of NBD1 where it resides, but also proper assembly of NBD1 with neighbouring CFTR domains, and that correction of either of these defects is only partially effective $[4,88,89]$. However, when both defects were corrected individually either by introducing suppressor mutations or by using combinations of experimental corrector compounds, it was possible to restore folding, cell surface expression and function of $\Delta \mathrm{F} 508$ to near normal levels [88-90]. Hence, these studies provide an explanation for the limited efficacy of VX-809 monotherapy and indicate that combinations of next generation corrector compounds that target both defects may be required for effective restoration of CFTR function in patients carrying $\Delta \mathrm{F} 508$ and potentially other processing mutations.

\section{Progress with gene and cell therapy approaches for CF}

Besides CFTR modulators, gene therapy represents a causative approach to correct the mutated gene. While promising in its early days, several pitfalls, such as vector design, in vivo delivery to epithelial cells (mucus and glycocalix) and off-target effects (random integration), have hampered the efficient implementation of this therapeutic strategy to date. However, novel gene vectors (lentiviral and non-viral vectors) [91], the zinc-finger nuclease-, TALEN-based and CRISPR/Cas9-mediated gene-editing technologies [92, 93], supplementation of mRNA to the lung [94], and the possibility to differentiate normal, patient-specific pluripotent stem cells into airway epithelial cells $[95,96]$ have revitalised the field and could lead to novel promising gene-correcting therapies in CF patients, as summarised elsewhere [91].

\section{Alternative targets to counteract the basic ion transport defects in CF airways Epithelial $\mathrm{Na}^{+}$channels}

Inhibition of enhanced ENaC-mediated $\mathrm{Na}^{+}$absorption and/or activation of alternative $\mathrm{Cl}^{-}$channels in the airways remain attractive alternative approaches to compensate for CFTR malfunction and restore airway surface hydration and mucociliary clearance in all patients with CF irrespective of their CFTR genotypes (fig. 1). In fact, $\mathrm{ENaC}$ has long been considered as an alternative therapeutic target [97]. However, probably due to its low potency, short half-life and limited deposition in mucus-obstructed airways, inhalation therapy with the classical ENaC blocker amiloride did not show therapeutic benefits in CF patients with established lung disease [98]. In contrast, preventive amiloride treatment had significant therapeutic effects by reducing mucus obstruction, airway inflammation and mortality in mice with CF-like lung disease [99]. These studies provided a proof of concept that inhaled amiloride may represent an effective preventive therapy if started in infants with CF who can now be diagnosed early by newborn screening [28]. While attractive as a strategy that may delay or even prevent irreversible lung damage in CF, this preventive treatment paradigm remains to be tested in clinical trials. At the same time, efforts are ongoing that focus on strategies for durable ENaC inhibition to improve efficacy in chronic CF airways disease. These efforts include: 1) the design of more potent and long-acting ENaC blockers [100, 101]; 2) inhibition of ENaCactivating proteases [44, 102]; 3) ENaC silencing with specific short interfering RNAs [103]; and 4) highcontent screens to identify novel ENaC regulators that may be druggable in CF lung disease [104]. Since $\mathrm{ENaC}$ is also expressed in the kidney, where it plays an important role in electrolyte balance, strategies are warranted that reduce systemic absorption of ENaC inhibitors effectively to prevent renal exposure and electrolyte imbalance including hyperkalaemia [105]. Several promising compounds are currently in active preclinical to early clinical development and may become available for clinical testing in patients with CF in the near future.

\section{Alternative $\mathrm{Cl}^{-}$channels}

Even before the discovery of CFTR, it was well established that airway epithelia express alternative $\mathrm{Ca}^{2+}$ activated $\mathrm{Cl}^{-}$channels (CaCC) that function normally and may, therefore, be exploited therapeutically to compensate for CFTR dysfunction in patients with CF (fig. 1) [106, 107]. This concept is supported by studies which showed that nucleotide-mediated activation of $\mathrm{CaCC}$ via purinergic $\left(\mathrm{P}_{2} \mathrm{Y}_{2}\right.$ receptormediated) signalling improves ASL height and mucus transport in primary CF airway epithelia [108]. However, efforts to activate CaCC indirectly using the long-acting $\mathrm{P}_{2} \mathrm{Y}_{2}$ receptor agonist denufosol failed to improve lung function or reduce exacerbations in CF patients in recent trials [109]. Furthermore, the development of drugs that activate $\mathrm{CaCC}$ directly has been hampered by the fact that its molecular identity remained elusive for many years. Recently, important progress was made in this field and several CaCC candidates were reported. While some of the proposed candidates, such as the CLCA family member hCLCA1/mCLCA3, could not be confirmed as CaCC in native airway epithelia [110], it is now clear that the transmembrane protein 16A (TMEM16A, also known as anoctamin-1) constitutes a $\mathrm{Cl}^{-}$channel that contributes to $\mathrm{Ca}^{2+}$-dependent $\mathrm{Cl}^{-}$secretion in human and mouse airways $[111,112]$. In addition, emerging data suggest SLC26A9, a member of the SLC26 family of anion transporters, as a promising alternative $\mathrm{Cl}^{-}$ channel in CF. As mentioned previously, recent studies in SLC26A9 knockout mice demonstrated that 
SLC26A9-mediated $\mathrm{Cl}^{-}$secretion is activated in airway inflammation and is essential to prevent airway mucus obstruction in the presence of mucus hypersecretion [52, 53]. Furthermore, a functional single nucleotide polymorphism (rs2282430) in the $3^{\prime}$ untranslated region of SLC26A9 that reduced protein expression by creating a binding site for a microRNA (has-miRNA-632) was shown to be associated with asthma in children, and SLC26A9 variants were recently detected in patients with non-CF bronchiectasis [113]. These results suggest SLC26A9 as a modifier and novel therapeutic target in CF and potentially other mucostatic airway diseases, including severe asthma and COPD. Of note, SLC26A9 was also found to contribute to the risk of meconium ileus in CF in a recent genome-wide association study [114]. The high throughput screens of large chemical libraries, developed to identify compounds that rescue mutant CFTR $[59,61]$, are now available for the discovery of drugs that open TMEM16A and SLC26 $\mathrm{A}^{-} \mathrm{Cl}^{-}$channels directly without altering cytosolic $\mathrm{Ca}^{2+}$, and test their potential as therapeutic targets to counteract deficient $\mathrm{Cl}^{-}$secretion and airway surface dehydration in $\mathrm{CF}$.

\section{CFTR dysfunction in non-epithelial cells}

While the pathogenetic role of CFTR dysfunction in CF lung disease has been studied most extensively in the superficial airway epithelium and submucosal glands, emerging evidence supports the notion that nonepithelial cell types in the lung, such as innate and adaptive immune cells and fibroblasts, also express CFTR $[115,116]$. Importantly, myeloid cells, i.e. neutrophils and macrophages, that accumulate in CF airways and are thought to drive chronic inflammation have been shown to express CFTR mRNA and protein and may utilise CFTR for proper phagolysosomal function and intracellular bacterial killing [117-119]. While our current understanding predicts that CFTR malfunction in airway epithelia is critical for triggering the disease, emerging studies with conditional deletion of CFTR in myeloid cells in mice [120] and studies with morpholino knock-down technology in zebrafish models [121] support the notion that CFTR dysfunction in myeloid cells may contribute to the pathogenesis and outcome of P. aeruginosa infection in CF lung disease. Beyond phagocytes, a potential role of CFTR in innate immunity includes other immune cell types, such as dendritic cells and natural killer T-cells, a topic discussed in more depth previously [115]. Furthermore, recent studies in CF mice and pigs suggest that CFTR malfunction may be implicated in abnormal development of cartilaginous airways that may contribute to early airflow obstruction, air trapping and respiratory dysfunction in CF [122-124].

\section{CFTR, cigarette smoke and COPD}

Given the key role of CFTR in proper mucociliary clearance and airway defence, the obvious question arises as to whether insights from CFTR research can be transferred to other more common chronic mucostatic lung diseases, including COPD, which has emerged as one of the most common chronic diseases in adults and the fourth leading cause of death worldwide [125]. COPD shares key phenotypical features with CF including reduced mucociliary clearance, small airways mucus obstruction, goblet cell metaplasia and mucus hypersecretion, chronic neutrophilic airway inflammation, and chronic bacterial infections [126, 127]. While it is well established that the majority of COPD is caused by cigarette smoke, the understanding of its pathogenesis remains limited. Interestingly, studies analysing the effect of cigarette smoke on airway ion transport demonstrated that cigarette smoke impairs CFTR expression and function in airway epithelia at various levels, ranging from reduced transcription to protein degradation, and resulting in "acquired" CFTR dysfunction (fig. 1) [128-130]. These studies demonstrated that cigarette smoke has acute effects on CFTR activity in the airways of healthy nonsmokers, causing $\sim 60 \%$ inhibition of CFTR-mediated $\mathrm{Cl}^{-}$secretion in vivo, and causes airway surface dehydration and reduced mucus transport in vitro. Recent evidence also suggests that smokers who developed COPD exhibit reduced CFTR-mediated $\mathrm{Cl}^{-}$secretion in their upper and lower airways, and that deficient ion transport is associated with airway mucus dehydration $[129,131,132]$. These results indicate that reduced levels of CFTR function in smokers may be insufficient for proper mucus hydration in the presence of cigarette smoke-induced goblet cell metaplasia and mucin hypersecretion, and may thus put smokers at risk for mucus stasis and chronic airways disease (fig. 1) [48, 50]. Moreover, recent studies found an association between CFTR expression, ceramide accumulation and severity of emphysema in COPD patients [133]. In addition, studies in mice showed that cigarette smoke decreases CFTR levels in lipid rafts, and that CFTR is implicated in apoptotic and autophagic responses in cigarette smoke-induced lung epithelial injury [134]. Interestingly, recent results indicate that effects of cigarette smoke on CFTR are not limited to the lungs, but that smoking causes systemic CFTR dysfunction, probably via a circulating agent, which may contribute to extrapulmonary disorders such as pancreatitis, male infertility and cachexia that have been linked to cigarette smoking and are also characteristic features of CF [135]. Collectively, these studies suggest that reduced levels of functional CFTR in smokers may contribute to COPD pathogenesis and that drugs developed to improve mutant CFTR function in CF may also be beneficial in patients with COPD. As a proof of concept, recent studies showed that the potentiator ivacaftor improved wild-type CFTR function and counteracted the effects of cigarette smoke on CFTR-mediated $\mathrm{Cl}^{-}$secretion, ASL height and mucus transport in 
human bronchial epithelial cells in vitro [130]. However, clinical trials are required to evaluate the therapeutic potential of CFTR modulators in COPD.

\section{Conclusions and unresolved questions}

The discovery of CFTR 25 years ago enabled CF researchers to unravel the molecular and cellular pathogenesis of CF. Basic and translational studies, including heterologous cell lines, human primary airway epithelia and animal models, have led to a detailed and still growing understanding of the function and regulation of CFTR. These studies demonstrate that reduced ASL volume and $\mathrm{pH}$ due to CFTR dysfunction are probable key pathogenetic mechanisms that cause early onset mucus plugging, trigger chronic neutrophilic inflammation and impair anti-bacterial host defence in CF lung disease. Clinical trials with the potentiator ivacaftor, one of the first CFTR modulators identified by high throughput screening, established an important proof of concept that effective mutation-specific CF pharmacotherapy is possible. This breakthrough constitutes a paradigm for the development of drugs for patients with other CFTR mutations. However, despite these major advances, we are still faced with unresolved questions and remaining challenges. For example, it remains uncertain how effectively misprocessing of the most common mutation $\Delta$ F508 can be corrected, and how much correction is required to yield clinical benefits in patients? How can we expand mutation-specific therapies to patients with rare CFTR mutations? Can rescue of mutant CFTR function reverse chronic airway inflammation and infection? How early do we need to start treatment with CFTR modulators to delay or even prevent chronic lung damage? To what extent can therapeutic targeting of $\mathrm{ENaC}$ and alternative $\mathrm{Cl}^{-}$channels compensate for CFTR dysfunction in vivo? Beyond $\mathrm{CF}$, how important is CFTR in the pathogenesis of other mucostatic airway diseases including COPD, and could this knowledge lead to more effective therapies for these common lung diseases? Therefore, basic and translational CF research remains essential to solve these important issues and make disease-modifying therapies available to all patients with CF, irrespective of their CFTR genotype or stage of disease.

\section{Acknowledgements}

We thank Peter-Michael Weber (Dept of Pediatrics, University of Tübingen, Tübingen, Germany) for excellent image design.

\section{References}

1 Rommens JM, Iannuzzi MV, Kerem B, et al. Identification of the cystic fibrosis gene: chromosome walking and jumping. Science 1989; 245: 1059-1065.

2 Riordan JR, Rommens JM, Kerem BS, et al. Identification of the cystic fibrosis gene: cloning and characterization of complementary DNA. Science 1989; 245: 1066-1072.

3 Kerem B, Rommens JM, Buchanan JA, et al. Identification of the cystic fibrosis gene: genetic analysis. Science 1989; 245: $1073-1080$.

Riordan JR. CFTR function and prospects for therapy. Annu Rev Biochem 2008; 77: 701-726.

5 Kreda SM, Mall M, Mengos A, et al. Characterization of wild-type and $\Delta$ F508 cystic fibrosis transmembrane regulator in human respiratory epithelia. Mol Biol Cell 2005; 16: 2154-2167.

6 Anderson MP, Gregory RJ, Thompson S, et al. Demonstration that CFTR is a chloride channel by alteration of its anion selectivity. Science 1991; 253: 202-205.

7 Smith JJ, Welsh MJ. cAMP stimulates bicarbonate secretion across normal, but not cystic fibrosis airway epithelia. J Clin Invest 1992; 89: 1148-1153.

8 Stutts MJ, Canessa CM, Olsen JC, et al. CFTR as a cAMP-dependent regulator of sodium channels. Science 1995; 269: 847-850.

9 Hopf A, Schreiber R, Mall M, et al. Cystic fibrosis transmembrane conductance regulator inhibits epithelial $\mathrm{Na}^{+}$ channels carrying Liddle's syndrome mutations. J Biol Chem 1999; 274: 13894-13899.

10 Knowles MR, Gatzy JT, Boucher RC. Increased bioelectric potential difference across respiratory epithelia in cystic fibrosis. N Engl J Med 1981; 305: 1489-1495.

11 Boucher RC, Stutts MJ, Knowles MR, et al. $\mathrm{Na}^{+}$transport in cystic fibrosis respiratory epithelia. Abnormal basal rate and response to adenylate cyclase activation. J Clin Invest 1986; 78: 1245-1252.

12 Mall M, Bleich M, Greger R, et al. The amiloride inhibitable $\mathrm{Na}^{+}$conductance is reduced by CFTR in normal but not in cystic fibrosis airways. J Clin Invest 1998; 102: 15-21.

13 Sheppard DN, Rich DP, Ostedgaard LS, et al. Mutations in CFTR associated with mild-disease-form $\mathrm{Cl}^{-}$channels with altered pore properties. Nature 1993; 362: 160-164.

14 Welsh MJ, Smith AE. Molecular mechanisms of CFTR chloride channel dysfunction in cystic fibrosis. Cell 1993; 73 : $1251-1254$.

15 Sosnay PR, Siklosi KR, Van Goor F, et al. Defining the disease liability of variants in the cystic fibrosis transmembrane conductance regulator gene. Nat Genet 2013; 45: 1160-1167.

16 Matsui H, Grubb BR, Tarran R, et al. Evidence for periciliary liquid layer depletion, not abnormal ion composition, in the pathogenesis of cystic fibrosis airways disease. Cell 1998; 95: 1005-1015.

17 Mall M, Grubb BR, Harkema JR, et al. Increased airway epithelial $\mathrm{Na}^{+}$absorption produces cystic fibrosis-like lung disease in mice. Nat Med 2004; 10: 487-493.

18 Stoltz DA, Meyerholz DK, Pezzulo AA, et al. Cystic fibrosis pigs develop lung disease and exhibit defective bacterial eradication at birth. Sci Transl Med 2010; 2: 29-31.

19 Boucher RC. Airway surface dehydration in cystic fibrosis: pathogenesis and therapy. Annu Rev Med 2007; 58: 157-170. 
20 Mall MA. Role of cilia, mucus, and airway surface liquid in mucociliary dysfunction: lessons from mouse models. J Aerosol Med Pulm Drug Deliv 2008; 21: 13-24.

21 Clarke LL, Grubb BR, Yankaskas JR, et al. Relationship of a non-cystic fibrosis transmembrane conductance regulator-mediated chloride conductance to organ-level disease in Cftr (-/-) mice. Proc Natl Acad Sci USA 1994; 91: 479-483.

22 Zhou Z, Duerr J, Johannesson B, et al. The $\beta \mathrm{ENaC}$-overexpressing mouse as a model of cystic fibrosis lung disease. J Cyst Fibros 2011; 10: Suppl. 2, S172-S182.

23 Rogers CS, Stoltz DA, Meyerholz DK, et al. Disruption of the CFTR gene produces a model of cystic fibrosis in newborn pigs. Science 2008; 321: 1837-1841.

24 Sun X, Sui H, Fisher JT, et al. Disease phenotype of a ferret CFTR-knockout model of cystic fibrosis. J Clin Invest 2010; 120: 3149-3160.

25 Klymiuk N, Mundhenk L, Kraehe K, et al. Sequential targeting of CFTR by BAC vectors generates a novel pig model of cystic fibrosis. J Mol Med (Berl) 2012; 90: 597-608.

26 Livraghi-Butrico A, Kelly EJ, Klem ER, et al. Mucus clearance, MyD88-dependent and MyD88-independent immunity modulate lung susceptibility to spontaneous bacterial infection and inflammation. Mucosal Immunol 2012; 5: 397-408.

27 Mall MA, Graeber SY, Stahl M, et al. Early cystic fibrosis lung disease: role of airway surface dehydration and lessons from preventive rehydration therapies in mice. Int J Biochem Cell Biol 2014; 52: 174-179.

28 Grasemann H, Ratjen F. Early lung disease in cystic fibrosis. Lancet Respir Med 2013; 1: 148-157.

29 Zuelzer WW, Newton WA. The pathogenesis of fibrocystic disease of the pancreas: a study of 36 cases with special reference to the pulmonary lesions. Pediatrics 1949; 4: 53-69.

30 Mall MA, Harkema JR, Trojanek JB, et al. Development of chronic bronchitis and emphysema in $\beta$-epithelial $\mathrm{Na}^{+}$ channel-overexpressing mice. Am J Respir Crit Care Med 2008; 177: 730-742.

31 Wielpütz MO, Eichinger M, Zhou Z, et al. In vivo monitoring of cystic fibrosis-like lung disease in mice by volumetric computed tomography. Eur Respir J 2011; 38: 1060-1070.

32 Gehrig S, Duerr J, Weitnauer M, et al. Lack of neutrophil elastase reduces inflammation, mucus hypersecretion and emphysema, but not mucus obstruction, in mice with CF-like lung disease. Am J Respir Crit Care Med 2014; 189: 1082-1092.

33 Trojanek JB, Cobos-Correa A, Diemer S, et al. Airway mucus obstruction triggers macrophage activation and MMP12-dependent emphysema. Am J Respir Cell Mol Biol 2014 [In press DOI: 10.1165/rcmb.2013-0407OC].

34 Pezzulo AA, Tang XX, Hoegger MJ, et al. Reduced airway surface $\mathrm{pH}$ impairs bacterial killing in the porcine cystic fibrosis lung. Nature 2012; 487: 109-113.

35 Sun X, Olivier AK, Liang B, et al. Lung phenotype of juvenile and adult cystic fibrosis transmembrane conductance regulator-knockout ferrets. Am J Respir Cell Mol Biol 2014; 50: 502-512.

36 Chen JH, Stoltz DA, Karp PH, et al. Loss of anion transport without increased sodium absorption characterizes newborn porcine cystic fibrosis airway epithelia. Cell 2010; 143: 911-923.

37 Joo NS, Cho HJ, Khansaheb M, et al. Hyposecretion of fluid from tracheal submucosal glands of CFTR-deficient pigs. J Clin Invest 2010; 120: 3161-3166.

38 Sly PD, Gangell CL, Chen L, et al. Risk factors for bronchiectasis in children with cystic fibrosis. N Engl J Med 2013; 368: 1963-1970.

39 Voynow JA, Fischer BM, Zheng S. Proteases and cystic fibrosis. Int I Biochem Cell Biol 2008; 40: 1238-1245.

40 Belaaouaj A, McCarthy R, Baumann M, et al. Mice lacking neutrophil elastase reveal impaired host defense against gram negative bacterial sepsis. Nat Med 1998; 4: 615-618.

41 Shapiro SD, Goldstein NM, Houghton AM, et al. Neutrophil elastase contributes to cigarette smoke-induced emphysema in mice. Am J Pathol 2003; 163: 2329-2335.

42 Hartl D, Latzin P, Hordijk P, et al. Cleavage of CXCR1 on neutrophils disables bacterial killing in cystic fibrosis lung disease. Nat Med 2007; 13: 1423-1430.

43 Caldwell RA, Boucher RC, Stutts MJ. Neutrophil elastase activates near-silent epithelial $\mathrm{Na}^{+}$channels and increases airway epithelial $\mathrm{Na}^{+}$transport. Am J Physiol Lung Cell Mol Physiol 2005; 288: L813-L819.

44 Gaillard EA, Kota P, Gentzsch M, et al. Regulation of the epithelial $\mathrm{Na}^{+}$channel and airway surface liquid volume by serine proteases. Pflugers Arch 2010; 460: 1-17.

45 Gentzsch M, Dang H, Dang Y, et al. The cystic fibrosis transmembrane conductance regulator impedes proteolytic stimulation of the epithelial $\mathrm{Na}^{+}$channel. J Biol Chem 2010; 285: 32227-32232.

46 Haerteis S, Krappitz M, Bertog M, et al. Proteolytic activation of the epithelial sodium channel (ENaC) by the cysteine protease cathepsin-S. Pflugers Arch 2012; 464: 353-365.

47 Le Gars M, Descamps D, Roussel D, et al. Neutrophil elastase degrades cystic fibrosis transmembrane conductance regulator via calpains and disables channel function in vitro and in vivo. Am J Respir Crit Care Med 2013; 187: $170-179$.

48 Button B, Cai LH, Ehre C, et al. A periciliary brush promotes the lung health by separating the mucus layer from airway epithelia. Science 2012; 337: 937-941.

49 Dickey BF. Biochemistry. Walking on solid ground. Science 2012; 337: 924-925.

50 Fahy JV, Dickey BF. Airway mucus function and dysfunction. N Engl J Med 2010; 363: 2233-2247.

51 Lohi H, Kujala M, Makela S, et al. Functional characterization of three novel tissue-specific anion exchangers SLC26A7, -A8, and -A9. J Biol Chem 2002; 277: 14246-14254.

52 Anagnostopoulou P, Dai L, Schatterny J, et al. Allergic airway inflammation induces a pro-secretory epithelial ion transport phenotype in mice. Eur Respir J 2010; 36: 1436-1447.

53 Anagnostopoulou P, Riederer B, Duerr J, et al. SLC26A9-mediated chloride secretion prevents mucus obstruction in airway inflammation. J Clin Invest 2012; 122: 3629-3634.

54 Coakley RD, Grubb BR, Paradiso AM, et al. Abnormal surface liquid pH regulation by cultured cystic fibrosis bronchial epithelium. Proc Natl Acad Sci USA 2003; 100: 16083-16088.

55 Garcia MA, Yang N, Quinton PM. Normal mouse intestinal mucus release requires cystic fibrosis transmembrane regulator-dependent bicarbonate secretion. J Clin Invest 2009; 119: 2613-2622.

56 Gustafsson JK, Ermund A, Ambort D, et al. Bicarbonate and functional CFTR channel are required for proper mucin secretion and link cystic fibrosis with its mucus phenotype. J Exp Med 2012; 209: 1263-1272. 

saline. N Engl J Med 2006; 354: 241-250.

68 Elkins MR, Robinson M, Rose BR, et al. A controlled trial of long-term inhaled hypertonic saline in patients with cystic fibrosis. N Engl J Med 2006; 354: 229-240.

69 Fuchs HJ, Borowitz DS, Christiansen DH, et al. Effect of aerosolized recombinant human DNase on exacerbations of respiratory symptoms and on pulmonary function in patients with cystic fibrosis. The Pulmozyme Study Group. N Engl J Med 1994; 331: 637-642.

70 Ramsey BW, Pepe MS, Quan JM, et al. Intermittent administration of inhaled tobramycin in patients with cystic fibrosis. Cystic Fibrosis Inhaled Tobramycin Study Group. N Engl J Med 1999; 340: 23-30.

71 Becq F, Mall MA, Sheppard DN, et al. Pharmacological therapy for cystic fibrosis: from bench to bedside. $J$ Cyst Fibros 2011; 10: Suppl. 2, S129-S145.

72 Sermet-Gaudelus I. Ivacaftor treatment in patients with cystic fibrosis and the G551D-CFTR mutation. Eur Respir Rev 2013; 22: 66-71.

73 Clancy JP, Jain M. Personalized medicine in cystic fibrosis: dawning of a new era. Am J Respir Crit Care Med 2012; 186: 593-597.

$74 \mathrm{Yu} \mathrm{H}$, Burton B, Huang CJ, et al. Ivacaftor potentiation of multiple CFTR channels with gating mutations. J Cyst Fibros 2012; 11: 237-245.

75 Van Goor F, Yu H, Burton B, et al. Effect of ivacaftor on CFTR forms with missense mutations associated with defects in protein processing or function. J Cyst Fibros 2014; 13: 29-36.

76 Quinton P, Molyneux L, Ip W, et al. $\beta$-adrenergic sweat secretion as a diagnostic test for cystic fibrosis. Am J Respir Crit Care Med 2012; 186: 732-739.

77 Hirtz S, Gonska T, Seydewitz $\mathrm{HH}$, et al. CFTR $\mathrm{Cl}^{-}$channel function in native human colon correlates with the genotype and phenotype in cystic fibrosis. Gastroenterology 2004; 127: 1085-1095.

78 Roth EK, Hirtz S, Duerr J, et al. The $\mathrm{K}^{+}$channel opener 1-EBIO potentiates residual function of mutant CFTR in rectal biopsies from cystic fibrosis patients. PLoS One 2011; 6: e24445.

79 Dekkers JF, Wiegerinck CL, De Jonge HR, et al. A functional CFTR assay using primary cystic fibrosis intestinal organoids. Nat Med 2013; 19: 939-945.

80 Esterly JR, Oppenheimer EH. Cystic fibrosis of the pancreas: structural changes in peripheral airways. Thorax 1968; 23: $670-675$.

81 Wielputz MO, Weinheimer O, Eichinger M, et al. Pulmonary emphysema in cystic fibrosis detected by densitometry on chest multidetector computed tomography. PLoS One 2013; 8: e73142.

82 Sly PD, Brennan S, Gangell C, et al. Lung disease at diagnosis in infants with cystic fibrosis detected by newborn screening. Am J Respir Crit Care Med 2009; 180: 146-152.

83 Eichinger M, Optazaite DE, Kopp-Schneider A, et al. Morphologic and functional scoring of cystic fibrosis lung disease using MRI. Eur J Radiol 2012; 81: 1321-1329.

84 Subbarao P, Stanojevic S, Brown M, et al. Lung clearance index as an outcome measure for clinical trials in young children with cystic fibrosis. A pilot study using inhaled hypertonic saline. Am J Respir Crit Care Med 2013; 188: 456-460.

85 Stahl M, Joachim C, Blessing K, et al. Multiple breath washout is feasible in the clinical setting and detects abnormal lung function in infants and young children with cystic fibrosis. Respiration 2014; 87: 357-363.

86 Wielputz MO, Puderbach M, Kopp-Schneider A, et al. Magnetic resonance imaging detects changes in structure and perfusion, and response to therapy in early cystic fibrosis lung disease. Am J Respir Crit Care Med 2014; 189: 956-965.

87 Clancy JP, Rowe SM, Accurso FJ, et al. Results of a phase IIa study of VX-809, an investigational CFTR corrector compound, in subjects with cystic fibrosis homozygous for the F508del-CFTR mutation. Thorax 2012; 67: 12-18.

88 Mendoza JL, Schmidt A, Li Q, et al. Requirements for efficient correction of $\Delta$ F508 CFTR revealed by analyses of evolved sequences. Cell 2012; 148: 164-174.

89 Rabeh WM, Bossard F, Xu H, et al. Correction of both NBD1 energetics and domain interface is required to restore $\Delta$ F508 CFTR folding and function. Cell 2012; 148: 150-163.

90 Okiyoneda T, Veit G, Dekkers JF, et al. Mechanism-based corrector combination restores $\Delta$ F508-CFTR folding and function. Nat Chem Biol 2013; 9: 444-454.

91 Griesenbach U, Alton EW. Moving forward: cystic fibrosis gene therapy. Hum Mol Genet 2013; 22: R52-R58.

92 Lee CM, Flynn R, Hollywood JA, et al. Correction of the $\Delta$ F508 mutation in the cystic fibrosis transmembrane conductance regulator gene by zinc-finger nuclease homology-directed repair. Biores Open Access 2012; 1: 99-108. 
93 Schwank G, Koo BK, Sasselli V, et al. Functional repair of CFTR by CRISPR/Cas9 in intestinal stem cell organoids of cystic fibrosis patients. Cell Stem Cell 2013; 13: 653-658.

94 Kormann MS, Hasenpusch G, Aneja MK, et al. Expression of therapeutic proteins after delivery of chemically modified mRNA in mice. Nat Biotechnol 2011; 29: 154-157.

95 Schmeckebier S, Mauritz C, Katsirntaki K, et al. Keratinocyte growth factor and dexamethasone plus elevated cAMP levels synergistically support pluripotent stem cell differentiation into alveolar epithelial type II cells. Tissue Eng Part A 2013; 19: 938-951.

96 Wong AP, Bear CE, Chin S, et al. Directed differentiation of human pluripotent stem cells into mature airway epithelia expressing functional CFTR protein. Nat Biotechnol 2012; 30: 876-882.

97 Mall MA. Role of the amiloride-sensitive epithelial $\mathrm{Na}^{+}$channel in the pathogenesis and as a therapeutic target for cystic fibrosis lung disease. Exp Physiol 2009; 94: 171-174.

98 Pons G, Marchand MC, d'Athis P, et al. French multicenter randomized double-blind placebo-controlled trial on nebulized amiloride in cystic fibrosis patients. The Amiloride-AFLM Collaborative Study Group. Pediatr Pulmonol 2000; 30: 25-31.

99 Zhou Z, Treis D, Schubert SC, et al. Preventive but not late amiloride therapy reduces morbidity and mortality of lung disease in $\beta E N a C$-overexpressing mice. Am J Respir Crit Care Med 2008; 178: 1245-1256.

100 Hirsh AJ, Zang J, Zamurs A, et al. Pharmacological properties of 552-02, a novel epithelial sodium channel blocker with potential clinical efficacy for cystic fibrosis lung disease. J Pharmacol Exp Ther 2008; 325: 77-88.

101 Hunt T, Atherton-Watson HC, Axford J, et al. Discovery of a novel chemotype of potent human ENaC blockers using a bioisostere approach. Part 1: quaternary amines. Bioorg Med Chem Lett 2012; 22: 929-932.

102 Garcia-Caballero A, Rasmussen JE, Gaillard E, et al. SPLUNC1 regulates airway surface liquid volume by protecting ENaC from proteolytic cleavage. Proc Natl Acad Sci USA 2009; 106: 11412-11417.

103 Gianotti A, Melani R, Caci E, et al. Epithelial sodium channel silencing as a strategy to correct the airway surface fluid deficit in cystic fibrosis. Am J Respir Cell Mol Biol 2013; 49: 445-452.

104 Almaca J, Faria D, Sousa M, et al. High-content siRNA screen reveals global ENaC regulators and potential cystic fibrosis therapy targets. Cell 2013; 154: 1390-1400.

105 O'Riordan TG, Donn KH, Hodsman P, et al. Acute hyperkalemia associated with inhalation of a potent $\mathrm{ENaC}$ antagonist: phase 1 trial of GS-9411. J Aerosol Med Pulm Drug Deliv 2014; 27: 200-208.

106 Anderson MP, Welsh MJ. Calcium and cAMP activate different chloride channels in the apical membrane of normal and cystic fibrosis epithelia. Proc Natl Acad Sci 1991; 88: 6003-6007.

107 Knowles MR, Clarke LL, Boucher RC. Activation by extracellular nucleotides of chloride secretion in the airway epithelia of patients with cystic fibrosis. N Engl J Med 1991; 325: 533-538.

108 Tarran R, Button B, Picher M, et al. Normal and cystic fibrosis airway surface liquid homeostasis. The effects of phasic shear stress and viral infections. J Biol Chem 2005; 280: 35751-35759.

109 Ratjen F, Durham T, Navratil T, et al. Long term effects of denufosol tetrasodium in patients with cystic fibrosis. J Cyst Fibros 2012; 11: 539-549.

110 Mundhenk L, Johannesson B, Anagnostopoulou P, et al. mCLCA3 does not contribute to calcium-activated chloride conductance in murine airways. Am J Respir Cell Mol Biol 2012; 47: 87-93.

111 Caputo A, Caci E, Ferrera L, et al. TMEM16A, a membrane protein associated with calcium-dependent chloride channel activity. Science 2008; 322: 590-594.

112 Rock JR, O'Neal WK, Gabriel SE, et al. Transmembrane protein 16A (TMEM16A) is a Ca2 ${ }^{+}$-regulated $\mathrm{Cl}^{-}$secretory channel in mouse airways. J Biol Chem 2009; 284: 14875-14880.

113 Bakouh N, Bienvenu T, Thomas A, et al. Characterization of SLC26A9 in patients with CF-like lung disease. Hum Mutat 2013; 34: 1404-1414.

114 Sun L, Rommens JM, Corvol H, et al. Multiple apical plasma membrane constituents are associated with susceptibility to meconium ileus in individuals with cystic fibrosis. Nat Genet 2012; 44: 562-569.

115 Hartl D, Gaggar A, Bruscia E, et al. Innate immunity in cystic fibrosis lung disease. J Cyst Fibros 2012; 11: 363-382.

116 Huaux F, Noel S, Dhooghe B, et al. Dysregulated proinflammatory and fibrogenic phenotype of fibroblasts in cystic fibrosis. PLoS One 2013; 8: e64341.

117 Di A, Brown ME, Deriy LV, et al. CFTR regulates phagosome acidification in macrophages and alters bactericidal activity. Nat Cell Biol 2006; 8: 933-944.

118 Zhou Y, Song K, Painter RG, et al. Cystic fibrosis transmembrane conductance regulator recruitment to phagosomes in neutrophils. J Innate Immun 2013; 5: 219-230.

119 Painter RG, Valentine VG, Lanson NA Jr, et al. CFTR expression in human neutrophils and the phagolysosomal chlorination defect in cystic fibrosis. Biochemistry 2006; 45: 10260-10269.

120 Bonfield TL, Hodges CA, Cotton CU, et al. Absence of the cystic fibrosis transmembrane regulator (CFTR) from myeloid-derived cells slows resolution of inflammation and infection. J Leukoc Biol 2012; 92: 1111-1122.

121 Phennicie RT, Sullivan MJ, Singer JT, et al. Specific resistance to Pseudomonas aeruginosa infection in zebrafish is mediated by the cystic fibrosis transmembrane conductance regulator. Infect Immun 2010; 78: 4542-4550.

122 Bonvin E, Le Rouzic P, Bernaudin JF, et al. Congenital tracheal malformation in cystic fibrosis transmembrane conductance regulator-deficient mice. J Physiol 2008; 586: 3231-3243.

123 Meyerholz DK, Stoltz DA, Namati E, et al. Loss of cystic fibrosis transmembrane conductance regulator function produces abnormalities in tracheal development in neonatal pigs and young children. Am J Respir Crit Care Med 2010; 182: 1251-1261.

124 Adam RJ, Michalski AS, Bauer C, et al. Air trapping and airflow obstruction in newborn cystic fibrosis piglets. Am J Respir Crit Care Med 2013; 188: 1434-1441.

125 Vestbo J, Hurd SS, Agusti AG, et al. Global strategy for the diagnosis, management, and prevention of chronic obstructive pulmonary disease: GOLD executive summary. Am J Respir Crit Care Med 2013; 187: 347-365.

126 Smaldone GC, Foster WM, O'Riordan TG, et al. Regional impairment of mucociliary clearance in chronic obstructive pulmonary disease. Chest 1993; 103: 1390-1396.

127 Hogg JC, Timens W. The pathology of chronic obstructive pulmonary disease. Annu Rev Pathol 2009; 4: 435-459.

128 Cantin AM, Hanrahan JW, Bilodeau G, et al. Cystic fibrosis transmembrane conductance regulator function is suppressed in cigarette smokers. Am J Respir Crit Care Med 2006; 173: 1139-1144. 
129 Clunes LA, Davies CM, Coakley RD, et al. Cigarette smoke exposure induces CFTR internalization and insolubility, leading to airway surface liquid dehydration. FASEB J 2012; 26: 533-545.

130 Sloane PA, Shastry S, Wilhelm A, et al. A pharmacologic approach to acquired cystic fibrosis transmembrane conductance regulator dysfunction in smoking related lung disease. PLoS One 2012; 7: e39809.

131 Dransfield MT, Wilhelm AM, Flanagan B, et al. Acquired CFTR dysfunction in the lower airways in COPD. Chest 2013; 144: 498-506.

132 Hill DB, Vasquez PA, Mellnik J, et al. A biophysical basis for mucus solids concentration as a candidate biomarker for airways disease. PLoS One 2014; 9: e87681.

133 Bodas M, Min T, Mazur S, et al. Critical modifier role of membrane-cystic fibrosis transmembrane conductance regulator-dependent ceramide signaling in lung injury and emphysema. J Immunol 2011; 186: 602-613.

134 Bodas M, Min T, Vij N. Critical role of CFTR-dependent lipid rafts in cigarette smoke-induced lung epithelial injury. Am J Physiol Lung Cell Mol Physiol 2011; 300: L811-L820.

135 Raju SV, Jackson PL, Courville CA, et al. Cigarette smoke induces systemic defects in cystic fibrosis transmembrane conductance regulator function. Am J Respir Crit Care Med 2013; 188: 1321-1330. 